\title{
Vigilância Nutricional e Morbidade de Crianças Menores de 5 Anos numa Unidade Básica de Saúde: Análise da Série Histórica 1987-91
}

\author{
Nutritional Surveillance and Morbidity of Children under 5 Years of Age \\ Seen in a Primary Health Care Unit: Historical Series 1987-91
}

\author{
Inês R. R. de Castro ${ }^{1,2}$ \& Luiz Antonio dos Anjos²
}

\begin{abstract}
CASTRO, I. R. R. \& ANJOS, L. A. Nutritional Surveillance and Morbidity of Children under 5 Years of Age Seen in a Primary Health Care Unit: Historical Series 1987-91. Cad. Saúde Públ., Rio de Janeiro, 9 (supplement 1): 36-45, 1993.
\end{abstract}

This paper presents preliminary analysis of the nutritional status assessment (NS) and morbidity of low-income children under 5 years of age seen by the Food and Nutritional Surveillance System of a primary health care unit in Rio de Janeiro between 1987 and 1991. The distribution of lengh/stature for age (LSA) was skewed to the left as early as the first semester of life. Approximately $20 \%$ of the children had values of body mass for age (BMA) below the $10^{\text {th }}$ percentile of the reference growth curve (NCHS). The prevalence of stunting ( $Z L S A \leq-2)$ decreased yearly during the period. The eight most frequent causes of visit to the unit were the same but the frequencies varied from year to year. In the last three years upper respiratory $(U R D)$ and digestive diseases $(D D)$ and undernutrition prevailed. NS did not correlate with $U P D$ but the prevalence of $D D$ was inversely associated with BMA percentile. These data indicate relevant growth deficits in the children which justifies the implementation of nutritional surveillance in health units. It is also clear that morbidity and NS must be correlated in future studies, so that, the role of nutritional surveillance is enhanced in the Health Sector.

Key words: Nutritional Surveillance; Morbidity; Child Development; Health Centers; Child Nutrition

\section{INTRODUÇÃO}

O Sistema de Vigilância Alimentar e Nutricional (SISVAN) envolve a coleta, o processamento e a análise de dados de grupos populacionais de forma contínua, com vistas à obtenção de um diagnóstico atualizado da situação alimentar e nutricional, bem como de suas tendências temporais, para subsidiar e avaliar intervenções (Mason et al., 1984).

Em maio de 1987 iniciou-se, no Centro de Saúde Escola Germano Sinval Faria (CSEGSF),

\footnotetext{
${ }^{1}$ Secretaria Municipal de Saúde do Rio de Janeiro. Rua Afonso Cavalcanti, 455/823. Rio de Janeiro, RJ, 20211110, Brasil.

${ }^{2}$ Escola Nacional de Saúde Pública. Rua Leopoldo Bulhões, 1480. Rio de Janeiro, RJ, 21041-210, Brasil.
}

unidade básica de saúde da Escola Nacional de Saúde Pública da Fundação Oswaldo Cruz, a implantação do SISVAN para crianças menores de 5 anos de idade, com o objetivo de monitorar seu perfil nutricional, a partir do crescimento, e de morbidade, a partir dos motivos de consulta. Desde então, diariamente são coletados, de todas as crianças consultadas, os seguintes dados registrados pelos profissionais de saúde atendentes: idade, sexo, massa corporal (MC), comprimento ou estatura, e motivos de consulta.

Este trabalho apresenta uma análise preliminar dos dados de crescimento e de motivos de consulta obtidos pelo SISVAN entre 1987 e 1991 com o objetivo de sistematizar uma experiência no nível local de atenção, visando contribuir para uma discussão sobre as possibi- 
lidades e limitações do SISVAN na rede de saúde.

\section{MATERIAL E MÉTODOS}

Os dados aqui apresentados dizem respeito a todas as crianças menores de 5 anos de idade atendidas entre maio de 1987 e dezembro de 1991 no CSEGSF, unidade básica de saúde localizada em Manguinhos, zona norte do município do Rio de Janeiro, cuja população adscrita é de aproximadamente 30.000 pessoas, em sua maioria moradores de favelas.

Os dados de número do prontuário, data de nascimento, data de consulta, sexo, área de moradia, massa corporal, comprimento ou estatura, e motivos de consulta foram copiados dos prontuários por estagiários de nutrição e residentes de saúde pública para uma planilha utilizada na informatização. Os dados de motivo de consulta foram codificados em grandes grupos, a partir de uma adaptação da Classificação Internacional de Problemas de Saúde em Atenção Primária (CIPSAP, 1986): puericultura, vacinação, doenças infecto-parasitárias, desnutrição/obesidade, anemia (a partir de dosagem de hemoglobina sérica), doenças do aparelho digestivo, doenças do aparelho respiratório, doenças de pele, distúrbios oculares e distúrbios do aparelho genito-urinário.

Por terem sido considerados todos os motivos de consulta registrados, e não apenas a queixa ou diagnóstico principal, o número total de motivos de consulta foi maior que o número de crianças atendidas.

Após terem sido armazenados em planilha eletrônica, os dados foram ordenados pelo nú- mero do prontuário e data da consulta, determinando-se a primeira visita mensal e anual para cada mês e ano. Para se obter as freqüências do estado nutricional anual, utilizou-se a primeira consulta do ano. As frequiências expressas por mês do ano foram calculadas a partir da primeira consulta do mês.

$\mathrm{O}$ estado nutricional (EN) foi determinado comparando-se os valores antropométricos das crianças (massa corporal e estatura ou comprimento) com a população de referência norteamericana (NCHS, 1977), recomendada pela Organização Mundial da Saúde (WHO, 1978; 1986) e pelo Ministério da Saúde para o acompanhamento do crescimento de crianças menores de 5 anos na rede de saúde (MS/INAN/ INAMPS, 1984). Para tanto, utilizou-se o programa de computador produzido pelo Center for Disease Control, dos Estados Unidos da América.

Foram gerados três indicadores: massa corporal para idade (MC/I), estatura (ou comprimento) para idade $(\mathrm{E} / \mathrm{I})$ e massa corporal para estatura (ou comprimento) (MC/E). Os valores encontrados para esses indicadores foram expressos em percentis e número de desvios padrões em relação à mediana (valor $Z$ ). Devido à falta de informações antropométricas, houve variações nos números de casos, os quais são apresentados nas tabelas.

\section{RESULTADOS}

Durante o período estudado houve aproximadamente 35 mil consultas, obtendo-se uma média de três consultas por criança/ano (Tabela 1). Nas primeiras consultas mensais houve uma média de 1,5 motivos de consulta (Tabela 2).

TABELA 1. Número de Consultas por Ano, Média de Consultas Anuais por Criança e Freqüência de Primeiras Visitas Anuais e Mensais para cada Ano de Crianças < 5 Anos Atendidas no CSEGSF entre 1987 e 1991

\begin{tabular}{lcccc}
\hline \hline Ano & Número de Consultas & $\begin{array}{c}\mathrm{N}^{\mathrm{o}} \text { de } 1^{\text {as }} \text { Consultas no } \\
\text { Mês }\end{array}$ & $\begin{array}{c}\mathrm{N}^{\mathrm{o}} \text { de } \\
1^{\text {as }} \text { Consultas no } \\
\text { Ano }\end{array}$ & $\begin{array}{c}\text { Média de Consultas } \\
\text { por Criança/Ano }\end{array}$ \\
\hline 1987 & 6566 & 5007 & 2469 & 2,66 \\
1988 & 8578 & 6965 & 2991 & 2,87 \\
1989 & 5090 & 4052 & 1990 & 2,56 \\
1990 & 7307 & 5481 & 2062 & 3,54 \\
1991 & 7384 & 5683 & 2092 & 3,53 \\
\hline Totais & 34925 & 27188 & 11604 & 3,01 \\
\hline \hline
\end{tabular}


TA B L A 2. Número de Consultas, Motivos de Consultas (Mot.) e Média de Motivos de Consultas de Crianças < 5 Anos Atendidas no CSEGSF entre 1987 e 1991

\begin{tabular}{lccc}
\hline \hline & $\mathrm{N}^{\mathrm{o}}$ de $1^{\text {as }}$ Consultas no & & \\
Ano & Mês & $\begin{array}{c}\mathrm{N}^{\text {o }} \text { de Mot. na } \\
1^{\text {a }} \text { Consulta no Mês }\end{array}$ & Média de Mot. \\
\hline 1987 & 5007 & 6722 & 1,34 \\
1988 & 6965 & 10307 & 1,48 \\
1989 & 4052 & 6414 & 1,58 \\
1990 & 5481 & 8256 & 1,51 \\
1991 & 5683 & 8819 & 1,55 \\
\hline Totais & 27188 & 40518 & 1,49 \\
\hline \hline
\end{tabular}

\section{Crescimento e Estado Nutricional}

A distribuição decilar do indicador massa corporal para idade (MC/I), para todas as faixas etárias e anos, indica que $20,4 \%$ das crianças encontram-se abaixo do primeiro decil, o que corresponde a duas vezes o esperado (Tabela $3)$. Utilizando-se as faixas de percentil recomendadas pelo Ministério da Saúde para o SISVAN (INAN, 1991), observa-se uma freqüência de $8,67 \%$ abaixo do Percentil 3 e de $11,73 \%$ entre os Percentis 3 e 10, dados que são 2,89 e 1,68 vezes maiores que o esperado para as respectivas faixas de distribuição (Tabela 3 ).

TABELA 3. Freqüência Percentual segundo o Indicador Massa Corporal para Idade, por Faixas de Percentis Selecionadas e Ano, na Primeira Consulta do Ano de Crianças < 5 Anos Atendidas no CSEGSF entre 1987 e 1991

\begin{tabular}{|c|c|c|c|c|}
\hline \multirow[b]{2}{*}{ Ano } & \multicolumn{3}{|c|}{ Percentis } & \multirow[b]{2}{*}{ Total $(\mathrm{N}$} \\
\hline & $\leq \mathrm{P}_{3}$ & $\mathrm{P}_{3} \dashv \mathrm{P}_{10}$ & $>\mathrm{P}_{10}$ & \\
\hline 1987 & 7,45 & 11,16 & 81,39 & 2133 \\
\hline 1988 & 9,56 & 10,73 & 79,71 & 2917 \\
\hline 1989 & 9,54 & 11,36 & 79,10 & 1971 \\
\hline 1990 & 9,26 & 12,11 & 78,63 & 2031 \\
\hline 1991 & 7,54 & 13,29 & 79,17 & 2069 \\
\hline Total & 8,67 & 11,73 & 79,60 & 11121 \\
\hline enc./esp. & 2,89 & 1,68 & 0,88 & - \\
\hline
\end{tabular}

* Proporção encontrada na clientela dividida pela proporção esperada na população de referência: 37 e $90 \%$ para valores abaixo de P3, entre P3 e P10 e maior que P10, respectivamente.
A distribuição decilar de MC/I por faixa etária é bastante semelhante em todos os anos. Desta forma, na média dos cinco anos, apresenta desvio para a esquerda em relação à referência já no segundo semestre de vida, o que se agrava com o aumento da idade (Figura 1).

Em relação ao indicador massa corporal para estatura (MC/E), a distribuição decilar por faixa etária mostrou-se semelhante à da população de referência, mudando, entretanto, de um leve desvio para a direita (Figura 2A), no primeiro semestre de vida, para um desvio para a esquerda, após o primeiro ano de vida (Figuras $2 \mathrm{C}$ e 2D). A distribuição dos valores $Z$ deste indicador é apresentada na Tabela 4. A frequiência de wasting $(z-2)$ e sobrepeso $(Z 2)$ ficou bem próxima dos valores da população de referência, variando de 2 a 3,8\%, dependendo do ano.

TA B E L A 4. Distribuição dos Valores Z do Indicador Massa Corporal para Estatura, por Ano, na Primeira Consulta do Ano de Crianças < 5 Anos Atendidas no CSEGSF entre 1987 e 1991

\begin{tabular}{lccccc}
\hline \hline \multicolumn{5}{c}{ Ano } \\
\hline Valor Z & 1987 & 1988 & 1989 & 1990 & 1991 \\
\hline & $(2321)^{*}$ & $(2511)$ & $(1686)$ & $(1805)$ & $(1753)$ \\
$\leq-2$ & 2,1 & 3,1 & 2,1 & 2,8 & 2,0 \\
$-1,9-1,9$ & 94,1 & 94,0 & 95,3 & 94,2 & 95,6 \\
$\geq 2$ & 3,8 & 2,9 & 2,6 & 3,0 & 2,4 \\
\hline \hline
\end{tabular}

* (n)

Quanto ao indicador estatura ou comprimento para idade $(E / I)$, houve um persistente desvio acentuado à esquerda, observado já nos primeiros seis meses de vida, que se manteve ou mesmo aumentou nas faixas etárias seguintes (Figura 3). 
FIG U R A 1. Distribuição Decilar da Massa Corporal para Idade em Função da Faixa Etária da Primeira Consulta do Ano de Crianças < 5 Anos Atendidas no CSE GSF entre 1987 e 1991
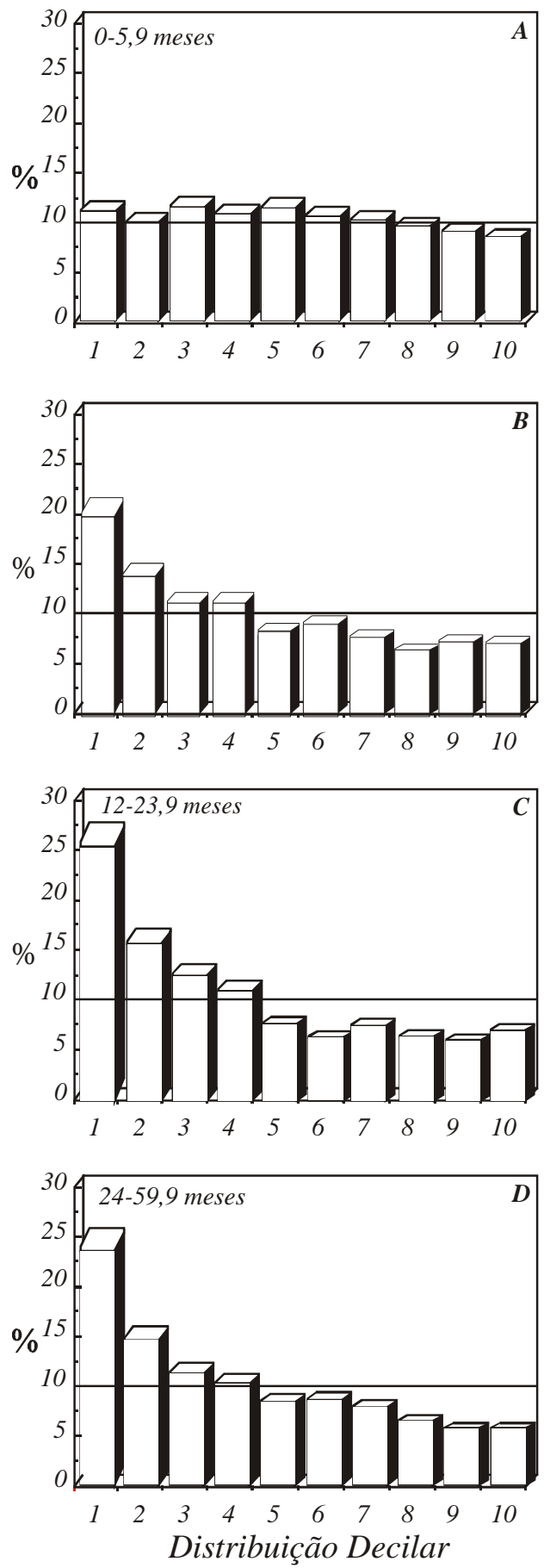

Dados são médias dos cinco anos. A linha horizontal indica o valor esperado de $10 \%$.
F I G U R A 2. Distribuição Decilar da Massa Corporal para Comprimento/Estatura em Função da Faixa Etária da Primeira Consulta do Ano de Crianças < 5 Anos Atendidas no CSEGSF entre 1987 e 1991
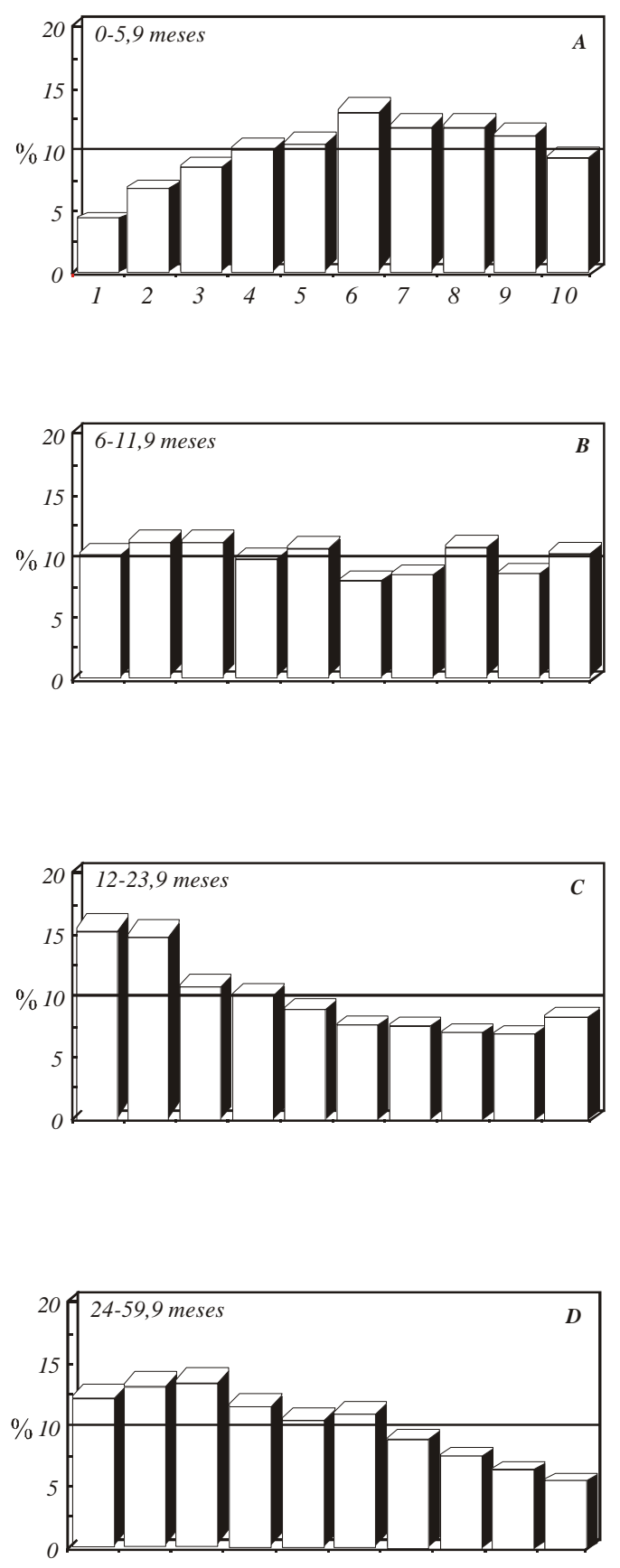

Distribuição Decilar

Dados são médias dos cinco anos. A linha horizontal indica o valor esperado de $10 \%$. 
Em relação às freqüências mensal (Figura 4) e anual (Tabela 5) de crianças abaixo de -2 desvios padrões segundo este indicador, pode-se observar uma tendência de aumento no ano de 1989 e de redução nos anos posteriores, particularmente em 1991. Esta flutuação foi observada em todas as faixas etárias, sendo menos nítida nas crianças de 0-5,9 meses (Tabela 5).

FIGURA 4. Frequiência Percentual de Valores $Z \leq$ -2 de Comprimento/Estatura para Idade da Primeira Consulta Mensal de Crianças < 5 anos Atendidas no CSEGSF entre 1987 e 1991, em Função do Mês e Ano de Atendimento

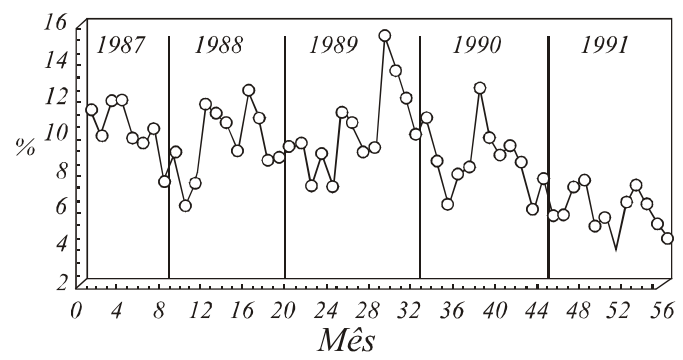

TA BELA 5. Frequiência Percentual de Crianças $<5$ Anos Atendidas no CSEGSF entre 1987 e 1991 com Valor Z de Estatura para Idade Abaixo de -2 por Faixa Etária e Ano

\begin{tabular}{lccccc}
\hline \hline $\begin{array}{l}\text { Faixa } \\
\text { Etária } \\
\text { (meses) }\end{array}$ & & \multicolumn{5}{c}{ Ano } \\
& 1987 & 1988 & 1989 & 1990 & 1991 \\
\hline $0 \dashv 5,9$ & 7,19 & 7,33 & 7,23 & 6,23 & 5,20 \\
$6 \dashv 11,9$ & 6,65 & 6,85 & 8,72 & 7,61 & 5,56 \\
$12 \dashv 23,9$ & 11,28 & 11,54 & 13,71 & 9,36 & 7,69 \\
$24 \dashv 59,9$ & 9,33 & 6,84 & 10,41 & 9,45 & 7,66 \\
\hline Total & 8,90 & 8,00 & 9,70 & 8,30 & 6,80 \\
\hline \hline
\end{tabular}

\section{Morbidade}

Os oito motivos de consulta mais freqüentemente registrados foram os mesmos de ano para ano, havendo, entretanto, grande variação em suas freqüências (Tabela 6). Nos últimos 3 anos, as doenças do aparelho respiratório, a desnutrição e as doenças do aparelho digestivo apareceram, nesta ordem, sistematicamente como os três principais motivos de demanda ao serviço.
FIG URA 3. Distribuição Decilar do Comprimento/Estatura para Idade em Função da Faixa Etária da Primeira Consulta do Ano de Crianças < 5 Anos Atendidas no CSEGSF entre 1987 e 1991
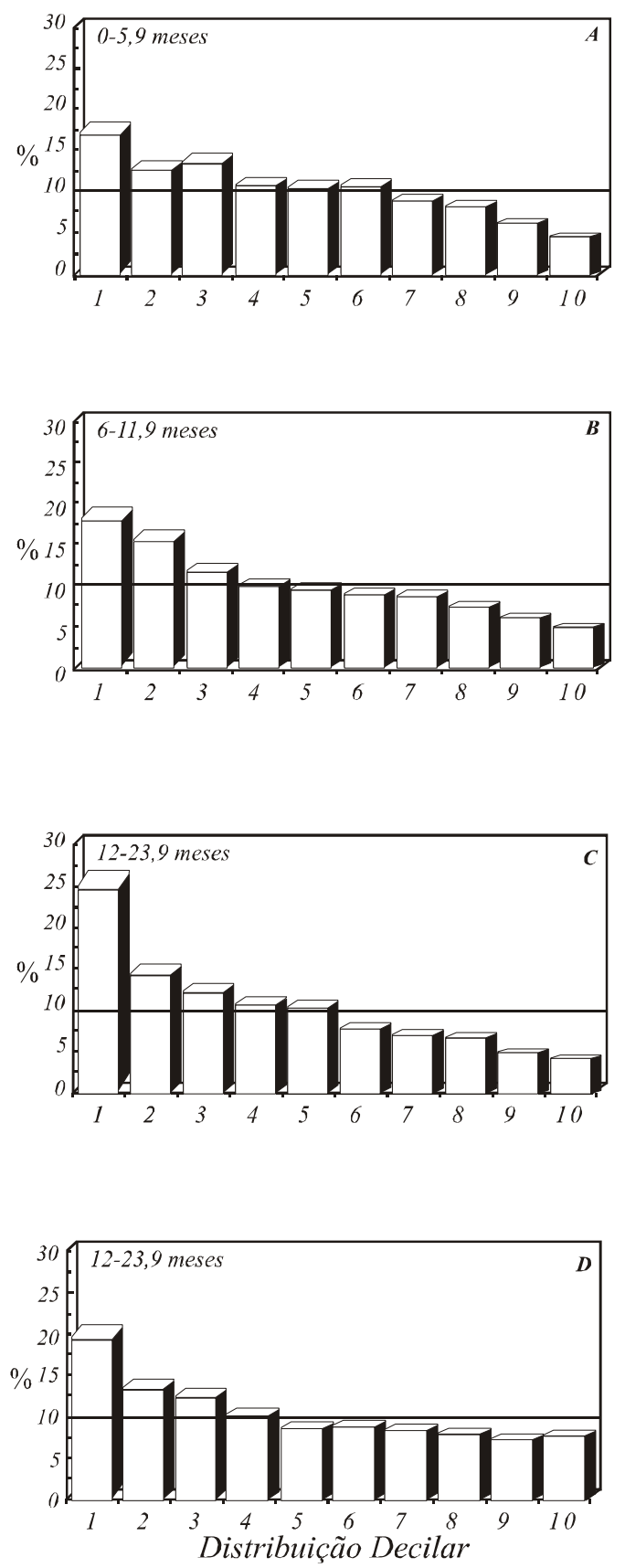

Dados são médias dos cinco anos. A linha horizontal indica o valor esperado de $10 \%$. 
TA BELA 6. Freqüência Percentual de Motivos de Consulta no Primeiro Atendimento do Ano de Crianças < 5 Anos Atendidos no CSEGSF entre 1987 e 1991

\begin{tabular}{|c|c|c|c|c|c|}
\hline \multirow{2}{*}{$\begin{array}{l}\text { Motivo } \\
\text { de } \\
\text { Consultas }\end{array}$} & \multicolumn{5}{|c|}{ Ano } \\
\hline & 1987 & 1988 & 1989 & 1990 & 1991 \\
\hline Puericultura & $\begin{array}{l}29,2 \\
(1)\end{array}$ & $\begin{array}{l}27,3 \\
(2)\end{array}$ & $\begin{array}{l}11,1 \\
(6)\end{array}$ & $\begin{array}{l}8,6 \\
(6)\end{array}$ & $\begin{array}{l}9,4 \\
(6)\end{array}$ \\
\hline $\begin{array}{l}\text { Aparelho } \\
\text { Respiratório }\end{array}$ & $\begin{array}{l}27,1 \\
(2)\end{array}$ & $\begin{array}{c}36,2 \\
(1)\end{array}$ & $\begin{array}{c}46,7 \\
(1)\end{array}$ & $\begin{array}{c}46,5 \\
(1)\end{array}$ & $\begin{array}{c}48,2 \\
(1)\end{array}$ \\
\hline Imunização & $\begin{array}{c}26,5 \\
(3)\end{array}$ & $\begin{array}{c}21,5 \\
(3)\end{array}$ & $\begin{array}{l}9,1 \\
(7)\end{array}$ & $\begin{array}{l}2,0 \\
(8)\end{array}$ & $\begin{array}{l}4,2 \\
(7)\end{array}$ \\
\hline $\begin{array}{l}\text { Aparelho } \\
\text { Digestivo }\end{array}$ & $\begin{array}{c}11,3 \\
(4)\end{array}$ & $\begin{array}{c}12,7 \\
(7)\end{array}$ & $\begin{array}{c}20,3 \\
(3)\end{array}$ & $\begin{array}{l}18,0 \\
(3)\end{array}$ & $\begin{array}{c}17,7 \\
(3)\end{array}$ \\
\hline $\begin{array}{l}\text { Infecto- } \\
\text { Para sitária }\end{array}$ & $\begin{array}{l}10,6 \\
(5)\end{array}$ & $\begin{array}{c}15,0 \\
(4)\end{array}$ & $\begin{array}{c}16,3 \\
(4)\end{array}$ & $\begin{array}{l}13,8 \\
(5)\end{array}$ & $\begin{array}{l}14,8 \\
(5)\end{array}$ \\
\hline Pele & $\begin{array}{c}10,2 \\
(6)\end{array}$ & $\begin{array}{c}13,5 \\
(5)\end{array}$ & $\begin{array}{c}13,5 \\
(5)\end{array}$ & $\begin{array}{c}15,1 \\
(4)\end{array}$ & $\begin{array}{l}16,2 \\
(4)\end{array}$ \\
\hline Desnutrição & $\begin{array}{l}5,5 \\
(7)\end{array}$ & $\begin{array}{c}13,1 \\
(6)\end{array}$ & $\begin{array}{l}25,8 \\
(2)\end{array}$ & $\begin{array}{l}32,1 \\
(2)\end{array}$ & $\begin{array}{c}24,9 \\
(2)\end{array}$ \\
\hline Anemia & $\begin{array}{l}0,7 \\
(8) \\
\end{array}$ & $\begin{array}{l}3,2 \\
(8) \\
\end{array}$ & $\begin{array}{l}2,9 \\
(8) \\
\end{array}$ & $\begin{array}{l}3,1 \\
(7) \\
\end{array}$ & $\begin{array}{l}4,1 \\
(8) \\
\end{array}$ \\
\hline
\end{tabular}

* Ordem de frequiência no respectivo ano

O motivo de consulta puericultura passou do primeiro lugar, em 1987, para o sexto entre as oito causas de visita mais freqüentes. Já a anemia manteve-se sempre entre os últimos motivos, alcançando $4 \%$ no máximo.

A freqüência de consultas por queixa do aparelho respiratório variou de mês para mês, parecendo ter um pico nos meses de inverno, principalmente nos anos de 1990 e 1991 (Figura 5). Já a flutuação da ocorrência de queixas do aparelho digestivo pareceu se dar em ciclos maiores que um ano (Figura 6).

\section{Estado Nutricional X Morbidade}

Ao se fazer o cruzamento das faixas de percentil selecionadas $(P<=3, P$ 3-10 e $P>$ 10) para os indicadores $\mathrm{MC} / \mathrm{I}$ e $\mathrm{E} / \mathrm{I}$ com os principais motivos de consulta (doenças do aparelho respiratório e doenças do aparelho digestivo) para as primeiras consultas do mês em todos os anos, observou-se que, para os dois indicadores, não parece existir uma relação clara entre freqüência de doenças do aparelho respiratório e estado nutricional (Tabelas 7 e 8).

FIG URA 5. Frequência Percentual de Primeira Consulta Mensal por Queixa do Aparelho Respiratório de Crianças < 5 Anos Atendidas no CSEGSF entre 1987 e 1991, em Função do Mês e Ano de Atendimento

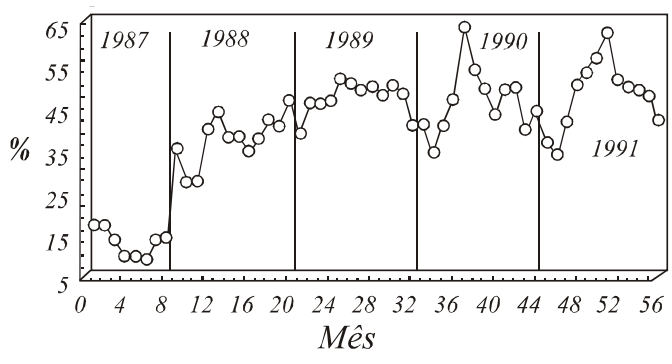

FIG URA 6. Freqüência Percentual de Primeira Consulta Mensal por Queixa do Aparelho Digestivo de Crianças < 5 Anos Atendidas no CSEGSF entre 1987 e 1991, em Função do Mês e Ano de Atendimento

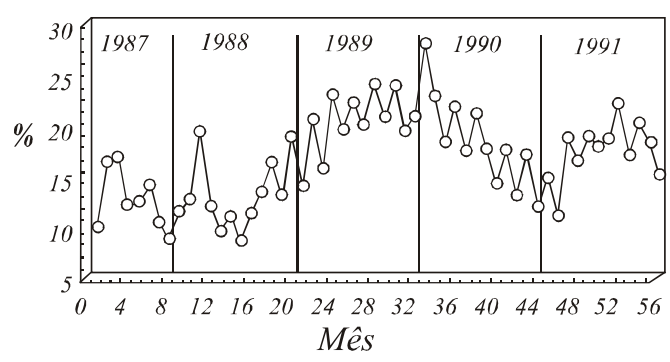

TABELA 7. Frequiência Percentual das Doenças do Aparelho Respiratório, por Ano e Faixa de Percentil do Indicador Massa Corporal para Idade, na Primeira Consulta do Mês de Crianças < 5 Anos Atendidas no CSEGSF entre 1987 e 1991

\begin{tabular}{lccc}
\hline \hline \multirow{3}{*}{ Ano } & \multicolumn{3}{c}{ Percentis } \\
\cline { 2 - 4 } & $\leq \mathrm{P}_{3}$ & $\mathrm{P}_{3} \dashv \mathrm{P}_{10}$ & $>\mathrm{P}_{10}$ \\
\hline 1987 & 21,79 & 25,96 & 25,88 \\
1988 & 42,16 & 35,40 & 35,63 \\
1989 & 49,74 & 46,04 & 46,35 \\
1990 & 47,98 & 45,31 & 46,61 \\
1991 & 45,72 & 44,96 & 49,07 \\
\hline Total & 41,48 & 39,53 & 40,71 \\
\hline \hline
\end{tabular}


TABELA 8. Freqüência Percentual das Doenças do Aparelho Respiratório, por Ano e Faixa de Percentil do Indicador Estatura para Idade, na Primeira Consulta do Mês de Crianças < 5 anos Atendidas no CSEGSF entre 1987 e 1991

\begin{tabular}{lccc}
\hline \hline & \multicolumn{3}{c}{ Percentis } \\
\cline { 2 - 4 } Ano & $\leq \mathrm{P}_{3}$ & $\mathrm{P}_{3} \dashv \mathrm{P}_{10}$ & $>\mathrm{P}_{10}$ \\
\hline 1987 & 21,81 & 20,68 & 26,11 \\
1988 & 36,74 & 37,92 & 33,88 \\
1989 & 45,81 & 50,24 & 46,06 \\
1990 & 41,76 & 45,72 & 47,37 \\
1991 & 41,61 & 48,86 & 49,97 \\
\hline Total & 37,55 & 40,68 & 40,68 \\
\hline \hline
\end{tabular}

Já em relação às doenças do aparelho digestivo, observa-se uma tendência de aumento da sua freqüência à medida que diminui a faixa de percentil para o indicador MC/I (Tabela 9), o mesmo não ocorrendo para o indicador E/I (Tabela 10).

Não se procedeu o cruzamento do indicador MC/E com os principais motivos de consulta, uma vez que se encontrou, na clientela estudada, uma distribuição deste indicador semelhante à da população de referência.

TABELA 9. Frequiência Percentual das Doenças do Aparelho Digestivo, por Ano e Faixa de Percentil do Indicador Massa Corporal para Idade, na Primeira Consulta do Mês de Crianças < 5 Anos Atendidas no CSEGSF entre 1987 e 1991

\begin{tabular}{lccc}
\hline \hline & \multicolumn{3}{c}{ Percentis } \\
\cline { 2 - 4 } Ano & $\leq \mathrm{P}_{3}$ & $\mathrm{P}_{3} \dashv \mathrm{P}_{10}$ & $>\mathrm{P}_{10}$ \\
\hline 1987 & 13,64 & 12,89 & 10,72 \\
1988 & 16,20 & 16,76 & 11,73 \\
1989 & 28,84 & 25,33 & 18,44 \\
1990 & 22,98 & 20,14 & 17,07 \\
1991 & 20,29 & 19,15 & 17,18 \\
\hline Total & 20,45 & 18,85 & 15,03 \\
\hline \hline
\end{tabular}

TA B E L A 10. Frequiência Percentual das Doenças do Aparelho Digestivo, por Ano e Faixa de Percentil do Indicador Estatura para Idade, na Primeira Consulta do Mês de Crianças < 5 Anos Atendidas no CSEGSF entre 1987 e 1991

\begin{tabular}{lccc}
\hline \hline & \multicolumn{3}{c}{ Percentis } \\
\cline { 2 - 4 } Ano & $\leq \mathrm{P}_{3}$ & $\mathrm{P}_{3} \dashv \mathrm{P}_{10}$ & $>\mathrm{P}_{10}$ \\
\hline 1987 & 10,61 & 12,70 & 11,60 \\
1988 & 13,17 & 11,52 & 12,89 \\
1989 & 27,54 & 21,26 & 19,99 \\
1990 & 18,88 & 16,15 & 18,31 \\
1991 & 19,58 & 19,16 & 19,10 \\
\hline Total & 19,79 & 17,02 & 17,57 \\
\hline \hline
\end{tabular}

\section{DISCUSSÃO}

O SISVAN, sendo um sistema de informações que contribui para o subsídio e a avaliação de intervenções, quando implementado em serviços de saúde, potencializa a prática de seus profissionais, pois, ao fornecer informações sobre perfil nutricional, introduz uma dimensão coletiva à análise da prática cotidiana, permitindo que o serviço se reorganize, melhorando a assistência prestada.

Os dados obtidos, por refletirem o perfil da demanda atendida e que foi registrado no prontuário pelo profissional atendente, fazem com que as interpretações e conclusões fiquem limitadas à situação da clientela atendida e às características de atendimento. Apesar destas limitações, as informações produzidas podem ser úteis na reorganização da atenção prestada.

Observando-se o indicador MC/I nas faixas de percentil recomendadas pelo Ministério da Saúde para o SISVAN (MS/INAN, 1991), merecem destaque as freqüências percentuais abaixo do primeiro decil: onde, pela referência, esperava-se encontrar $10 \%$ das crianças, encontrou-se 2,04 vezes o esperado; onde esperava-se encontrar 7\% (faixa entre os Percentis 3 e 10), encontrou-se 1,68 vezes o esperado; e onde esperava-se encontrar 3\% (faixa abaixo do Percentil 3, de maior risco de desnutrição), encontrou-se 2,89 vezes o esperado.

Partindo-se da constatação descrita acima e supondo-se que o SISVAN deva ser incorporado às práticas da rede de saúde (Arruda, 1992) 
e, portanto, aos programas de atenção integral já implantados, vale a pena refletir sobre a pertinência da inclusão da curva do Percentil 3 no "Cartão da Criança" — instrumento preconizado pelo Ministério da Saúde para o acompanhamento do crescimento de crianças menores de 5 anos na rede de saúde (MS/INAN/ INAMPS, 1984). Esta inclusão seria bastante interessante, pois, sem ser criado qualquer instrumento paralelo ao Cartão (pelo contrário, valorizando-o), seria possível triar crianças sob níveis diferentes de risco nutricional, podendose subsidiar, de maneira mais refinada, estratégias de acompanhamento na rede de saúde.

Observando-se o indicador E/I, causou surpresa encontrar uma tendência de diminuição da freqüência de crianças abaixo de -2 desvios padrões nos anos mais recentes. Embora trabalhos recentes tenham apontado a importante freqüência de crianças nesta situação (Monteiro, 1988; INAN, 1990; Monteiro et al., 1992), tais estudos não fornecem dados comparativos ano a ano, o que torna mais difícil a interpretação dos achados das crianças atendidas no CSEGSF.

De qualquer forma, as seguintes suposições podem ser levantadas: 1) por algum motivo, a unidade de saúde diminuiu sua captação e acompanhamento de crianças com maior risco de desnutrição crônica; 2) houve, de fato, algum impacto do monitoramento do estado nutricional no perfil deste indicador na clientela infantil atendida; e/ou 3) houve uma flutuação na prevalência deste indicador na população adscrita à unidade, por razões outras que não o monitoramento. Como não há indícios da primeira causa, sobram as duas últimas.

A continuação do SISVAN poderá melhor indicar se a tendência encontrada se confirma, sugerindo, talvez, alguma contribuição da Unidade de Saúde, a partir do SISVAN, na melhoria do quadro de desnutrição crônica das crianças de Manguinhos, através do atendimento diferenciado às crianças sob risco nutricional. Tal contribuição poderia se dar através de, por exemplo, um maior número de consultas com pediatra e/ou nutricionista, proporcionando um maior vínculo entre a criança, seu responsável e o serviço; orientação alimentar condizente com a faixa etária da criança, bem como com os hábitos e condições de vida da família; e detecção precoce de doenças, garantindo uma intervenção oportuna e evitando o agravamento das mesmas.

Em relação aos consolidados de motivos de consulta, vários aspectos merecem ser comentados. O fato de o motivo de consulta puericultura ter passado do primeiro (com frequiência superior a 30\%) para o sexto lugar (com freqüência inferior a $15 \%$ ) provavelmente está relacionado não só ao aumento do registro da desnutrição pelos profissionais que realizaram os atendimentos, mas também ao maior registro de motivos de consulta pelos codificadores, fato observado ao longo dos anos no processo de coleta de dados. Como, pela regra de codificação na planilha, o motivo de consulta puericultura só poderia ser concomitante ao motivo imunização, nunca podendo vir acompanhado de um agravo ou queixa, à medida que aumentou o registro destes últimos (Tabela 2), diminuiu o de puericultura.

Os altos percentuais de doenças do aparelho respiratório a partir de 1989 superaram a freqüência deste agravo tradicionalmente registrada nos serviços de atenção primária, que costuma ficar em torno de $30 \%$ a $40 \%$ (Alleyne et al., 1983). Isto provavelmente deveu-se ao fato de os codificadores do SISVAN coletarem informações sobre todos os motivos de consulta indicados no prontuário, e não só sobre os diagnósticos indicados pelos profissionais de saúde como sendo os principais, o que poderia caracterizar um sobre-registro de doenças do aparelho respiratório.

Em relação à anemia, agravo a ser também monitorado pelo SISVAN (INAN, 1991; Silva \& Castro, 1993), os poucos estudos realizados no Brasil evidenciam altas prevalências em crianças menores de 5 anos (Nogueira \& Brandim, 1992; Salzano et al., 1985; Szarfarc, 1985). Monteiro (1988), ao estudar este agravo (hemoglobina sérica $<11 \mathrm{~g} . \mathrm{dl}^{-1}$ ) entre crianças residentes no município de São Paulo, encontrou prevalências de $53,7,58,1$ e $35,6 \%$ para as faixas de 6-12, 12-24 e 0-60 meses, respectivamente.

No entanto, nos resultados obtidos no presente estudo, chamam a atenção os baixíssimos percentuais registrados, uma vez que a unidade de saúde em questão dispõe de laboratório próprio. Isto indica que este agravo provavel- 
mente tem passado despercebido — ou não está sendo valorizado, não sendo portanto registrado - pelos profissionais de saúde responsáveis pelo atendimento da clientela infantil.

Quanto aos resultados do cruzamento de estado nutricional e motivo de consulta, causou surpresa não serem encontradas tendências que demonstrassem a relação entre a frequiência de doenças do aparelho respiratório e o estado nutricional segundo o indicador MC/I, uma vez que estudos indicam uma estreita relação entre desnutrição e internações e mortes por estas doenças (Niobey, 1992; Victora et al., 1989).

Talvez esta tendência não possa mesmo ser observada com dados de um serviço de atenção básica ao se analisar o total de atendimentos por doenças do aparelho respiratório. Isto porque a codificação utilizada pode mascarar possíveis tendências, já que não se pode discriminar uma doença mais grave, como pneumonia, de uma infecção leve, sendo a primeira mais esperada de ocorrer em crianças desnutridas.

Some-se a isto o fato já relatado anteriormente de um possível sobre-registro de doenças do aparelho respiratório. Pode-se afirmar que, se este ocorreu, o foi independentemente do estado nutricional; logo, poder-se-ia argumentar, com pertinência, que esta atitude não introduziu nenhum viés. No entanto, supondo-se que as crianças desnutridas de fato apresentaram uma maior freqüência de doenças do aparelho respiratório, a proporção de sobre-registro seria menor entre elas e, obviamente, maior entre as eutróficas, o que dificultaria a observação de alguma relação entre estado nutricional e doenças do aparelho respiratório.

Já em relação ao cruzamento de doenças do aparelho digestivo e estado nutricional segundo o mesmo indicador citado acima, pode-se observar uma relação entre o aumento da frequiência e a diminuição da faixa de percentil. Também aqui nada se pode afirmar sobre possíveis diferenças na gravidade da ocorrência deste grupo de agravos de acordo com o estado nutricional.

Vale ressaltar que não se observou nenhuma tendência nos consolidados que cruzaram motivo de consulta com faixas de percentil segundo o indicador E/I, talvez pelas limitações da codificação dos agravos, talvez por não haver mesmo uma relação clara, já que este indicador reflete uma desnutrição crônica ou pregressa, que parece oferecer menos riscos de adoecer.

Além de indicarem um relevante quadro de déficit de crescimento da clientela estudada, reforçando a pertinência da implantação do SISVAN na rede de saúde, estes resultados também apontam para a necessidade de se realizarem mais estudos com dados de demanda relacionando perfil nutricional e perfil de morbidade da clientela. Esta articulação certamente potencializará o SISVAN, valorizando sua capacidade de monitoramento cotidiano dos perfis nutricional e de morbidade e sublinhando seu importante papel como instrumento de informações para a formulação e avaliação de intervenções na rede de saúde.

\section{AGRADECIMENTOS}

Os autores agradecem à Dra. Maria Tereza Costa pelos comentários de uma versão preliminar do artigo. Este trabalho contou com o apoio do CNPq, processos 503069/88-4 e 301076/89-8 .

\section{RESUMO}

CASTRO, I. R. R. \& ANJOS, L. A.

Vigilância Nutricional e Morbidade de

Crianças Menores de 5 Anos numa

Unidade Básica de Saúde: Análise da Série

Histórica 1987-91. Cad. Saúde Públ., Rio de Janeiro, 9 (suplemento 1): 36-45, 1993.

O presente estudo apresenta uma análise preliminar dos dados de estado nutricional (EN) e de morbidade de crianças menores de 5 anos, coletados pelo Sistema de Vigilância Alimentar e Nutricional (SISVAN) entre 1987 e 1991 numa unidade de atenção primária à saúde que atende a uma população favelada do Rio de Janeiro. Os perfis encontrados nas quase 35.000 consultas no período indicaram um importante desvio à esquerda, já no primeiro semestre de vida, para o indicador estatura/idade (E/I). Revelaram, ademais, que $20,4 \%$ das crianças apresentavam massa corporal para idade (MC/I) abaixo do percentil 10 da referência NCHS. Houve uma aparente tendência de diminuição da 
freqüência de crianças com nanismo (valor $Z$ $\mathrm{E} / \mathrm{I} \leq-2 Z$ ) ao longo dos anos. Os oito motivos de consulta mais freqüentes foram os mesmos durante o período, mas suas frequiências variaram de ano para ano. Nos últimos 3 anos, os motivos mais freqüentes foram doenças do aparelho respiratório (AR), doenças do aparelho digestivo (AD) e desnutrição. Não se observou uma tendência clara entre freqüência de doenças do AR e EN, observando-se, no entanto, uma relação inversa entre a freqüência de $\mathrm{AD}$ e o percentil de MC/I. Além de indicarem um relevante quadro de déficit de crescimento da clientela estudada, justificando a implantação do SISVAN, estes resultados também reforçam a necessidade de outros estudos relacionando os perfis nutricional e de morbidade da clientela antendida em serviços de atenção primária, potencializando o papel do SISVAN na rede de saúde.

Palavras-Chave: Vigilância Nutricional; Morbidade; Desenvolvimento Infantil; Centros de Saúde; Nutrição da Criança

\section{REFERÊNCIAS BIBLIOGRÁFICAS}

ALLEYNE, G. A. O.; LUELMO, F.; PLAUT, R. \& SCHMUNIS. G., 1983. A cute respiratory infections in children. Washington, DC: PAHO. (Pan American Health Organization Scientific Publication, no. 493)

ARRUDA, B. K. G., 1992. Sistema de Vigilância Alimentar e Nutricional. Frustrações, desafios e perspectivas. Cadernos de Saúde Pública, 8: 96-101.

CIPSAP, 1986. Clasificación Internacional de Problemas en A tención Primaria. Buenos Aires, Argentina: Centro Internacional para la Medicina Familiar.

INAN (Instituto Nacional de Alimentação e Nutrição), 1990. Resultados Preliminares da Pesquisa Nacional sobre Saúde e Nutrição. Brasília: Instituto Nacional de Alimentação e Nutrição.

MASON, J. B.; HABICHT, J. P.; TABATABAI, H. \& VALVERDE, V., 1984. Vigilancia Nutricional. Genebra: World Health Organization.

MONTEIRO, C. A., 1988. Saúde e Nutrição das Crianças de São Paulo. Diagnóstico, Contrastes Sociais e Tendências. São Paulo: Hucitec. MONTEIRO, C. A.; BENÍCIO, M. H. D. \& GOUVEIA, N. C., 1992. Saúde e nutrição das crianças brasileiras no final da década de 80 . In:
Perfil Estatístico de Crianças e Mães no Brasil - Aspectos de Saúde e Nutrição de Crianças no B rasil - 1989, pp. 19-42, Rio de Janeiro: IBGE/ UNICEF/INAN.

MS (Ministério da Saúde) \& INAN (Instituto Nacional de Alimentação e Nutrição), 1991. Sistema de Vigilância Alimentar e Nutricional - Proposta para A tuação a Nível Nacional. Brasília: Ministério da Saúde.

MS (Ministério da Saúde); INAN (Instituto Nacional de Alimentação e Nutrição); INAMPS (Instituto Nacional de Assistência Médica da Previdência Social), 1984. Acompanhamento do Crescimento e Desenvolvimento. Brasília: MS.

NCHS, 1977. NCHS Growth Curves for Children Birth-18 Y ears United States. Washington, DC: U.S. Printing Office. (Vital and Health Statistics Series 11, no. 165, DHEW Pub. no. 78-1650)

NIOBEY, F. M. L.; DUCHIADE, M. P.; VASCONCELOS, A. G. G.; CARVALHO, M. L.; LEAL, M. C. \& VALENTE, J. G., 1992. Fatores de risco para morte por pneumonia em menores de um ano em uma região metropolitana do Sudeste do Brasil. Um estudo tipo caso-controle. Revista de Saúde Pública, 26: 229-238.

NOGUEIRA, N. N. \& BRANDIM, R. F. R., 1992. Anemia por deficiência de ferro em crianças de 24 a 59 meses de idade no estado do Piauí em 91. In: Programa e Resumos do II Congresso Brasileiro de Epidemiologia. Rio de Janeiro: Associação Brasileira de Pós-Graduação em Saúde Coletiva.

SALZANO, A. C.; TORRES, M. A. A.; BATISTA FILHO, M. \& ROMANI, S. A. M., 1985. Anemias em crianças de dois serviços de saúde de Recife, PE (Brasil). Revista de Saúde Pública, 19: 499-507.

SZARFARC, S. C., 1985. Diagnóstico de deficiência de ferro na infância. Revista de Saúde Pública 19: 278-284.

SILVA, D. O. \& CASTRO, I. R. R., 1993. O Sistema de Vigilância Alimentar e Nutricional na Rede de Saúde - Manual para Implantação. Rio de Janeiro: CSEGSF/Ensp/Fiocruz.

VICTORA, C. G; BARROS, F. C. \& VAUGHAN, J. P., 1988. Epidemiologia da Desigualdade. São Paulo: Hucitec.

WHO (World Health Organization), 1978. A Growth Chart for International Use in Maternal and Child Health Care: Guidelines for Primary Health Care Personnel. Geneva, Switzerland: World Health Organization.

, 1986. Use and interpretation of anthropometric indicators of nutritional status. Bulletin of the World Health Organization, 64: 929-941. 